\title{
Development of an optical membrane for humidity
}

\section{Journal Article}

\section{Author(s):}

Mohr, Gerhard J.; Spichiger-Keller, Ursula E.

Publication date:

1998

Permanent link:

https://doi.org/10.3929/ethz-b-000422883

Rights / license:

In Copyright - Non-Commercial Use Permitted

Originally published in:

Microchimica Acta 130(1-2), https://doi.org/10.1007/BF01254587 


\title{
Development of an Optical Membrane for Humidity
}

\author{
Gerhard J. Mohr* and Ursula E. Spichiger-Keller \\ Centre for Chemical Sensors, ETH Technopark, Zurich, Switzerland
}

\begin{abstract}
N, N$-dioctylamino)-4'-trifluoroacetyl-azobenzene $\left(\mathrm{ETH}^{\mathrm{T}} 4001\right)$, together with the catalyst tridodecylmethylammonium chloride, is dissolved in the hydrophilic polymer polyurethane Tecoflex. The resulting membrane layers show high sensitivity toward water vapour and allow the application of the membranes for humidity measurements. Upon exposure to humid air, the membrane exhibits a decrease in absorbance at a wavelength around $490 \mathrm{~nm}$ and an increase at around $430 \mathrm{~nm}$. This signal change is caused by the conversion of the trifluoroacetyl group of the reactand into a diol, thus changing the electron delocalisation of the reactand. The sensor layer exhibits a dynamic range from $1 \%$ to $100 \%$ RH with highest sensitivity in the $5 \%-40 \% \mathrm{RH}$ range. The limit of detection is $0.5 \% \mathrm{RH}$. The amount of added catalyst enables the sensitive range to be tailored. The selectivity over ethanol and carbonate is sufficient for the membrane to be used for long-term measurements of air. The change in colour of the humidity-sensitive membrane from red to yellow also means it can be used as an optical test strip.
\end{abstract}

Key words: humidity-sensitive membrane, chromoReactand, ETH $^{\mathrm{T}} 4001$.

Monitoring humidity is important for both human comfort and in those industrial processes where water vapour might affect the quality of products. The humidity sensors available today are based on physical methods such as capacitance or resistance measurements of polymers and ceramics $[1,2]$. These sensors usually exhibit a linear sensitive range from 0 to $100 \%$

\footnotetext{
* To whom correspondence should be addressed
}

relative humidity (RH) with response times being in the range of around $5 \mathrm{~min}$. Their low drift means they can be used discontinuously over one year without recalibration. However, the sensor devices are expensive and are sometimes affected by exposure to liquid water or organic solvent vapours.

During the last decade, optical sensors have become increasingly important since they can potentially be miniaturized, they are relatively cheap and simple to set up, and they allow continuous and reversible measurements. Among these are optical sensors for humidity that rely on the reaction of metal ion complexes with water resulting in colour changes such as cobalt(II) chloride [3] or on luminescence changes in calcein or metallo-porphyrines $[4,5]$. Another approach is based on the response of polarity-sensitive dyes to humid gases $[6,7]$. Recently, a water sensor has been developed which is based on luminescence quenching in an osmium complex [8]. The phosphorescence of the luminophore allows the luminescence lifetime of the complex rather than its intensity to be measured. This can be done independent of bleaching or leaching of the indicator.

Trifluoroacetophenone derivatives have been reported to interact selectively with alcohols $[9,10]$ and humidity [11]. However, these derivatives have their wavelength of maximum absorbance at around $305 \mathrm{~nm}$ and they exhibit low absorption coefficients (typically around $6000 \mathrm{lmol}^{-1} \mathrm{~cm}^{-1}$ ). We have, therefore, recently developed the new chromogenic reactand $\mathrm{ETH}^{\mathrm{T}}$ 4001, which has comparable sensitivity and selectivity but with absorbance shifted by more than $200 \mathrm{~nm}$ into the visible spectral range $[12,13]$. The name "reactand" was coined, in analogy with the 
term "ligand", for the class of host compounds which react reversibly with an analyte or target compound [14]. In our research, the reactand was embedded in plasticizer-free polyurethane, together with the basic catalyst tridodecylmethylammonium chloride, and the resulting humidity-sensitive membranes were investigated in terms of sensitivity, selectivity, response, effect of catalyst, and operational/shelf lifetime.

\section{Experimental}

\section{Apparatus}

A gas flow system similar to the system presented in ref. 11 was designed. In essence, it included mixing humid air with dry air in well-defined proportions. The humid air was obtained by bubbling dry air through wash bottles filled with double-distilled water. The optical measurements were performed by securing the humiditysensitive layers in a flow-through cell located in the spectrophotometer and pumping air with different humidities at a flow rate of $1.21 \mathrm{~min}^{-1}$ through the cell. The relative humidity and temperature of the humid vapour was measured via a commetcial electronic hygrometer HP101A-P15 from Rotronic AG (Bassersdorf, Switzerland). The absorption spectra of the humidity-sensitive layers were recorded on a Uvikon 942 spectrophotometer from Kontron Instruments (Zürich, Switzerland) at $24 \pm 1{ }^{\circ} \mathrm{C}$. The ethanol gas concentrations were obtained by bubbling dry air through wash bottles containing aqueous solutions of ethanol. The ethanol concentrations in the gas phase were then calculated according to ref. 15.

\section{Materials}

All reagents were of analytical grade. The polyurethane Tecoflex EG $80 \mathrm{~A}$ was obtained from Thermedics Inc. (Woburn, MA). Tridodecylmethylammonium chloride (TDMACI) and dichloromethane (DCM) were from Fluka AG (Buchs, Switzerland). The synthesis of $\mathrm{ETH}^{\mathrm{T}} 4001$ has already been described in detail [13].

\section{Preparation of the Sensor Layers (see Table 1)}

Sensor membranes $M 1-M 3$ were obtained by dissolving $2.0 \mathrm{mg}$ of $\mathrm{ETH}^{\mathrm{T}} 4001$, the respective quantity of TDMACI $(0.44 \mathrm{mg}$ for $M 1$, $20 \mathrm{~mol} \%$ relative to the reactand; $4.4 \mathrm{mg}$ for $M 3,200 \mathrm{~mol} \%$ relative to the reactand) and $100 \mathrm{mg}$ of EG80A in $1.5 \mathrm{ml}$ of DCM. A dustfree glass plate was placed in a spin coating device with a DCMsaturated atmosphere $0.3 \mathrm{ml}$ of the solution was transferred onto the rotating glass support. Then, the resulting membranes were placed in ambient air for drying, upon which they gradually turned from red to orange/yellow in humid air. Before measurements, the membranes whose thickness was in the range of $2-5 \mu \mathrm{m}$ were conditioned in distilled water in order to activate the catalyst.

\section{Results}

\section{Choice of Reactand and Polymer}

The reactand, $\mathrm{ETH}^{\mathrm{T}} 4001$, is an azo dye which consists of the azobenzene chromophore, and a terminal alkylamino and trifluoroacetyl group. The alkylamino group acts as an electron donor and the octyl chains attached to the nitrogen atom render the reactand lipophilic and thus soluble in polymer materials. The trifluoroacetyl group of the reactand acts both as an electron acceptor and as an interactive site for nucleophilic species. The reaction of the trifluoroacetyl group with alcohols or water to form hemiacetals and diols, respectively, results in a significant decrease in its acceptor capacity and, therefore, in a blue-shift of the absorbance spectrum.

Tecoflex EG $80 \mathrm{~A}$, the matrix chosen in this study, is a thermoplastic aliphatic polyurethane which is synthesized from methylene bis(cyclohexyl)diisocyanate, poly(tetramethylene ether glycol), and 1,4butanediol. Consequently, the material exhibits relatively high hydrophilicity. This is shown in its high water uptake which, depending on the specification, can range from as small as $1 \%$ to over $1000 \%$. The present polymer exhibits a water uptake of around 3$5 \%$, which reduces swelling effects on the response. The material shows higher hydrophilicity than plasticized PVC, plasticized PVC being the matrix generally used in ISEs and optodes. In the present case, this hydrophilicity is thought to have a positive effect on the selectivity and sensitivity of the humidity

Table 1. Compositions and sensor characteristics of $M I-M 3$, composed of the reactand $\mathrm{ETH}^{\mathrm{T}} 4001$ and the polymer EG80A

\begin{tabular}{|c|c|c|c|c|c|}
\hline & TDMACI & $\overline{\mathrm{RSC}^{\mathrm{a}}}$ & $\operatorname{LOD}^{b}$ & $\begin{array}{l}\text { Forward/reverse } \\
\text { response (uncond.) }\end{array}$ & $\begin{array}{l}\text { Forward/reverse response } \\
\text { (cond.) }^{\mathrm{d}}\end{array}$ \\
\hline$M 1$ & $20 \mathrm{~mol} \%^{\mathrm{e}}$ & $80 \%$ & $0.5 \%$ & $3-4 h />4 h$ & $15-20 \mathrm{~min} / 30-50 \mathrm{~min}$ \\
\hline$M 2$ & $0 \mathrm{~mol} \%$ & $80 \%$ & $0.5 \%$ & $3-4 \mathrm{~b} />4 \mathrm{~h}$ & $3-4 h />4 h$ \\
\hline$M 3$ & $200 \mathrm{~mol} \%$ & $80 \%$ & $0.2 \%$ & $3-4 h />4 h$ & $10-15 \mathrm{~min} / 20-30 \mathrm{~min}$ \\
\hline
\end{tabular}

\footnotetext{
${ }^{2}$ RSC Relative signal change between nitrogen and water measured at $500 \mathrm{~nm}$.

b Limit of detection.

${ }^{c}$ Response of the unconditioned sensor membranes.

d Response of the membranes conditioned in distilled water prior to use.

e Amount of TDMACI relative to the reactand $\mathrm{ETH}^{\mathrm{T}} 4001$.
} 
sensors. In addition, the polyurethane exhibits good flexibility even without the use of a plasticizer. The omission of a plasticizer is generally advantageous because plasticizers evaporate at elevated temperatures and thus affect the shelf-lifetime. The mechanical stability of polyurethane membranes is superior to that of plasticized PVC membranes. Tecoflex is biocompatible and the fact that it is an aliphatic polyurethane implies that there are no carcinogenic by-products. The lack of aromatic moieties in the polymer also prevents yellowing, which is generally important in materials used in optical sensing.

\section{Sensor Response}

On exposure to dry air, membrane $M I$ exhibited an absorbance maximum at around $490 \mathrm{~nm}$ corresponding to the trifluoroacetyl form of the reactand. Exposure to humidified air resulted in a decrease in the absorbance at $490 \mathrm{~nm}$ and a new maximum was formed at around $430 \mathrm{~nm}$, which corresponded to the diol form of the reactand (Figs. 1 and 2). The relative signal change of $M 1$ when changing from 0 to $100 \%$ RH was as high as $80 \%$. The LOD was found to be $0.5 \% \mathrm{RH}$. The relative standard deviations for $1.9 \%$ and $84.5 \%$ RH $(\mathbf{n}=10)$ were determined to be $1.36 \%$ and $0.79 \%$, respectively. The forward response time $t_{95}$ (for $95 \%$ of the total signal change to occur) was in the range of $3-4 \mathrm{~h}$, whereas the time for the reverse response was in the range of $4 \mathrm{~h}$. In order to shorten the response time, the membrane was preconditioned in distilled water for $10 \mathrm{~min}$ in order to activate the catalyst by converting the tridodecylmethylammo-
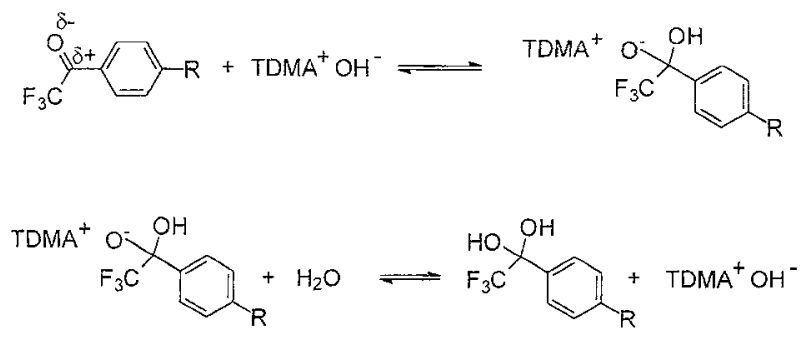

TDMA $^{+} \quad$ tridodecylmethylammonium cation

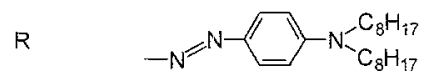

Fig. 1. Schematic representation of the proposed mechanism of the base-catalyzed recognition of humidity; see also ref. [11]. Only a small fraction of TDMACI is assumed to be converted into the hydroxide during conditioning in water

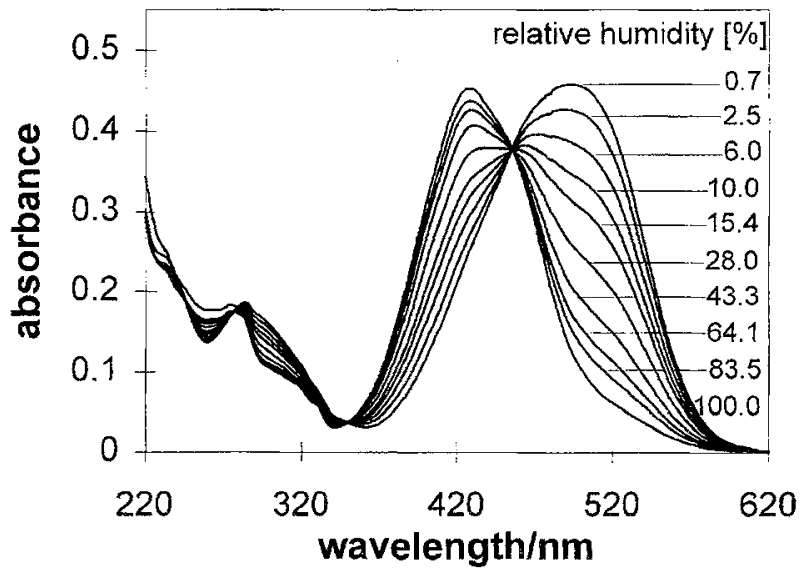

Fig. 2. Absorbance spectra of $M I\left(\mathrm{ETH}^{\mathrm{T}} 4001,20 \mathrm{~mol} \%\right.$ TDMACI) in contact with different concentrations of humid air. When changing from dry to humid air, the trifluoroacetyl group of the reactand $\mathrm{ETH}^{\mathrm{T}} 4001$ is converted into a diol. The chemical reaction is fully reversible

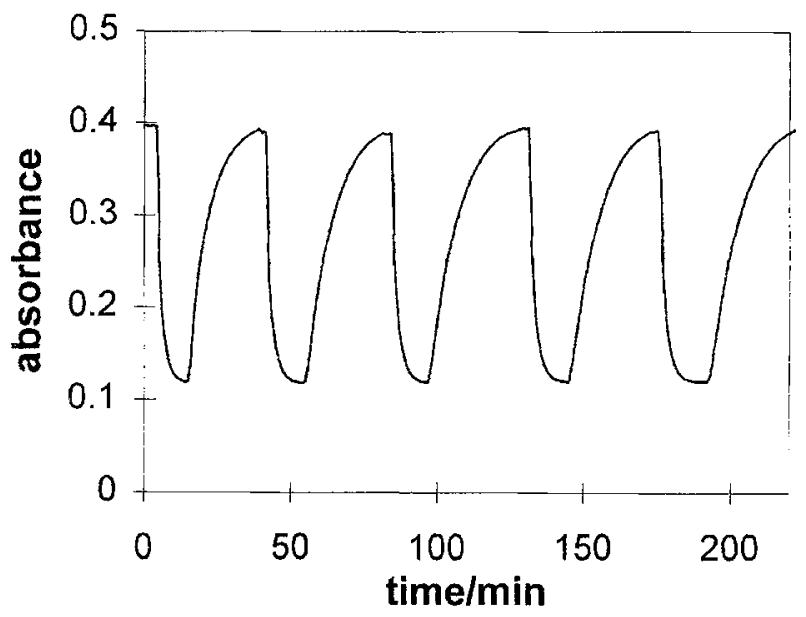

Fig. 3. Short time reproducibility of $M I$ on alternate exposure to $1.9 \%$ and $85.2 \% \mathrm{RH}$, measured at a wavelength of $500 \mathrm{~nm}$. The membrane $M I$ was used immediately after preparation and conditioning in distilled water for $10 \mathrm{~min}$ prior to use. The slope of the response indicates the continuous increase in response time

nium chloride into the respective hydroxide (Fig. 1, [11]). The forward response time, $t_{95}$, of the resulting sensor was in the range of 15-20 min, whereas the time for the reverse response was in the range of $30-$ 50 min (Fig. 3). However, this activation was found to decrease continuously and, after 2 weeks, the response times of $M I$ went down to reach the initial values of the unconditioned membrane.

In order to investigate the effect of TDMACI on the sensor response, membranes without TDMACI $(M 2)$ and with 200 mol\% of TDMACI (M3) were prepared. The presence of TDMACI turned out to affect both the sensitivity and response of the membranes. 


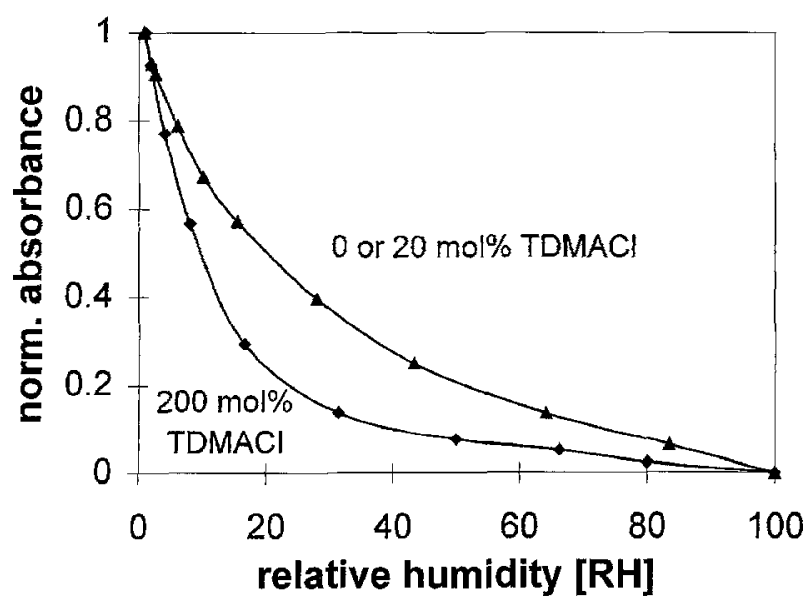

Fig. 4. Work functions of $M 1-M 3$ on exposure to air of different humidities, measured at a wavelength of $500 \mathrm{~nm}$. The value for $100 \%$ RH is obtained by immersing the membrane in water

Without TDMACI, the response time for changing between dry and humidified air was in the range of 3$4 \mathrm{~h}$ for both forward and reverse responses regardless of whether the membranes $M 2$ were preconditioned in water. Sensor membranes containing $20 \mathrm{~mol} \%$ TDMACI (MI) showed fast responses after preconditioning, whereas without preconditioning, the response times were similar to $M 2$. Pre-conditioned membranes $M 3$ containing $200 \mathrm{~mol} \%$ of TDMACI behaved comparable to membranes $M I$ in that they exhibited a fast response after preconditioning, and a slow response without preconditioning.

Whereas both $M 1$ and $M 2$ exhibited a similar sensitivity to humidified air, $M 3$ exhibited a significantly enhanced sensitivity with a LOD of $0.1 \% \mathrm{RH}$ (Figure 4). It is important to notice that in no case did preconditioning of the membrane in water affect the calibration plot. A compilation of the characteristics of $M 1-M 3$ is found in Table 1.

\section{Selectivity Toward Ethanol and Carbon Dioxide}

The hydrophilic polymer polyurethane, Tecoflex, used in this investigation, favors the extraction of water into the sensor membrane. Therefore, the sensor membrane should be relatively insensitive to ethanol and carbon dioxide.

$M I$ was exposed to $52 \mathrm{~Pa}$ ethanol at $25 \% \mathrm{RH}$ and $103 \mathrm{~Pa}$ ethanol at $50 \% \mathrm{RH}$. In both cases, the presence of ethanol did not have any effect on the relative signal changes. In order to investigate the cross- sensitivity to carbon dioxide, the sensor membrane M1 was exposed first to air of different RHs, and then to carbon dioxide of different RHs. No difference in the relative signal changes was observed, regardless of whether air or carbon dioxide was the carrier gas for the water vapour.

\section{Discussion}

Effects of Reactand and Polymer on the Performance of the Humidity-Sensitive Membranes

The reactand, $\mathrm{ETH}^{\mathrm{T}}$ 4001, embedded in the polyurethane Tecoflex shows a significant change in absorbance on exposure to humid air. This response is catalyzed by the presence of TDMACI (Fig. 1), but also takes place without a catalyst. This response is brought about by water nucleophilically reacting with the highly electrophilic trifluoroacetyl group to form a diol. The conversion of the trifluoroacetyl group into the diol changes the optical properties of the dye by shifting the maximum of absorbance from $490 \mathrm{~nm}$ to around $430 \mathrm{~nm}$.

The reactand, $\mathrm{ETH}^{\mathrm{T}}$ 4001, has already been used for sensing alcohols $[12,13]$. The reactand was placed together with the basic catalyst TDMACI in PVC plasticized by dioctylsebacate. The sensitivity of the alcohol sensor membrane was in the $1.0-50 \mathrm{vol} \%$ range with relative signal changes of around $50 \%$ for $50 \mathrm{vol} \%$ of ethanol. The response of the alcohol sensor was mainly governed by the lipophilicity of the alcohols, which indicates that the response was mainly caused by the extraction of alcohols into the polymer membrane, followed by the chemical reaction with the trifluoroacetyl group. Using the more polar plasticizer, ETH 8045, together with PVC as the polymer matrix, the relative signal changes of the sensor membrane on exposure to ethanol were reduced by a factor of two. This indicates that a more polar matrix disfavors the extraction of alcohols into the sensor membrane and decreases its sensitivity. Exchanging the matrix of the alcohol sensor (plasticized PVC) by polyurethane resulted in a membrane which, upon exposure to water, already underwent full conversion of the trifluoroacetyl group into the diol, and no further reaction with alcohols occurred. As a consequence, the use of the hydrophilic polyurethane favours the extraction of water and thus provides an appropriate matrix for a humidity sensor. 
Trifluoroacetophenone derivatives have also been used for sensing carbonates in ion-selective electrodes, relying on the interaction of the nucleophilic carbonate anion with the trifluoroacetyl group. We therefore investigated the response of $M l$ to carbon dioxide. However, no significant effect on the relative signal changes was observed when $M I$ was exposed to humid carbon dioxide instead of humid air [16].

The hydrophilic polymer polyurethane has a significant effect on the response times of the humidity sensor membranes. Due to the hydrophilicity of the polymer, there is a significant water uptake, which is converted into an optical signal by the reactand $\mathrm{ETH}^{\mathrm{T}}$ 4001. Since this water uptake is known to be relatively slow, the response times of the sensor membranes are slow as well, generally in the range of $3-4 \mathrm{~h}$. The slow response, however, is also due to the fact that the chemical reaction takes considerably more time than the physical interaction. The polar hydrophilic matrix also seems to affect the response time, since it is assumed that the catalyst slowly decomposes after conditioning in water (see next section). In the case of the alcohol sensors based on the unpolar matrix PVC and dioctylsebacate, a deterioration in the response similar to the humiditysensitive membranes is not observed. The alcohol sensors remain operationally stable for over one week without any significant decrease in response [13].

\section{Effect of the Additive on the Response}

The ion exchanger TDMACI has a significant effect on the response of the sensor membranes composed of the polyurethane polymer and $\mathrm{ETH}^{\mathrm{T}}$ 4001. As has already been shown for humidity sensors based on trifluoroacetyl derivatives [11], the response time is tremendously improved in the presence of TDMACI. Wang et al. [11] assumed that the catalytic effect of TDMACI is based on the partial exchange of chloride with hydroxide during preconditioning of the sensor membrane in water. The basic membrane environment is then thought to catalyze the conversion of the trifluoroacetyl group into a diol. They confirmed the assumption for an exchange of chloride against hydroxide during membrane conditioning by using tridodecylmethylammonium thiocyanate instead of chloride as the catalyst. Due to the lipophilicity of thiocyanate, the anion was not exchanged with hydroxide during conditioning in water and no catalytic effect was observed. However, there was no evidence that simple conditioning of the sensor membrane in water resulted in a conversion of the tridodecylmethylammonium chloride into the strongly basic and unstable hydroxide.

In the present work, a comparable behaviour of the sensor membranes is observed. Without TDMACI, the response is in the range of $3-4 \mathrm{~h}$, whereas in the presence of TDMACI, the response can be in the range of 15-30 min. Again, preconditioning of the sensor membranes in water is required to obtain this enhancement in response time. Unfortunately, the positive effect of the conditioning process is reversed after approximately two weeks and a slow response similar to the unconditioned membranes is observed again. We assume the slow decomposition of the catalyst tridodecylmethylammonium hydroxide to be the reason for this increase in response time. Sensor membranes that were stored in dry air for one month and then preconditioned in water responded fast to humidity. In contrast, membranes that have been preconditioned and then stored for one month exhibited a slow response time similar to that of the membranes without any catalyst. This suggests that, during the conversion of the tridodecylmethylammonium chloride into the basic hydroxide by conditioning in water, an unstable ammonium hydroxide is produced. It is known that alkylammonium hydroxides decompose via Hofmann degradation [17]. This decomposition reaction is not as effective with alkylammonium ions that exhibit ethyl or propyl groups instead of the methyl group in the tridodecylmethylammonium ion. We are confident, therefore, that we will be able to improve the stability of the sensor membranes by synthesizing and investigating more stable catalysts for the humidity sensor.

\section{Conclusion}

The membranes described here provide a simple means of determining relative humidity. They are composed of a non-plasticized bio-compatible polymer containing the reactand and a catalyst which allows the adjustment of the sensitivity. The response time of the membranes in the range of 3-4 hours (without conditioning) prevents their use in optical sensors where faster responses are required. However, these sensor membranes might find applications as probes for humidity. For this they are especially suitable since the reaction with water vapour is easily visible (colour change from red to yellow). Conse- 
quently, the membranes might be used as indicator test strips for materials that should not be exposed to humidity.

Acknowledgments. This work was supported by the Austrian Science Foundation through an Erwin-Schrödinger fellowship (J1260-CHE) which is gratefully acknowledged.

\section{References}

[1] N. Yamazoe, Y. Shimizu, Sens. Act. A 1986, 10, 377-398.

[2] H. Huang, P. K. Dasgupta, Anal. Chem. 1990, 62, 1935-1942.

[3] F. Boltinghouse, K. Abel, Anal. Chem. 1989, 61, 1863-1866.

[4] Y. Sadako, Y. Sakai, X. Wang, J. Mater. Sciences 1994, 29, 883-886.

[5] D. B. Papkovsky, G. V. Ponomarev, S. F. Chernov, A. N. Ovchinnikov, I. N. Kurochkin, Sens. Act. B 1994, 22, 57-61.

[6] Y. Sadaoka, M. Matsuguchi, Y. Sakai, Y. Murata, Chem. Lett. 1992, 53-56.

[7] T. E. Brook, M. N. Taib, R. Narayamaswamy, Sens. Act. B 1997, 38-39, 272-276.

[8] Q. Chang, Z. Murtaza, J. R. Lakowicz, G. Rao, Anal. Chim. Acta 1997, 350, 97-104.
[9] K. Seiler, K. Wang, M. Kuratli, W. Simon, Anal. Chim. Acta 1991, 244, 151-160.

[10] R. Wild, D. Citterio, J. Spichiger, U. E. Spichiger, J. Biotechnol. 1996, 50, 37-46.

[11] K. Wang, K. Seiler, J. P. Haug, B. Lehmann, S. West, K. Hartman, W. Simon, Anal. Chem. 1991, 63, 970-974.

[12] G. J. Mohr and U. E. Spichiger-Keller, Proc. SPIE 1997, 3105 , 130-137.

[13] G. J. Mohr, D. Citterio, and U. E. Spichiger-Keller, Sens. Act. $B$, 1998, 49, 224-232.

[14] U. E. Spichiger-Keller, Chemical Sensors and Biosensors for Medical and Biological Applications, Wiley VCH, Weinheim, 1998.

[15] J. Gmehling, U. Onken, in: Vapor-liquid equilibrium data collection. Aqueous-organic systems. (D. Behrens, R. Eckermann, eds.), Chemistry Data Series. Vol. 1, Part 1., DECHEMA, Deutsche Gesellschaft für Chemisches Apparatewesen, Frankfurt a. M., 1977.

[16] C. Behringer, B. Lehmann, J. P. Haug, K. Seiler, W. E. Morf, K. Hartman, W. Simon, Anal. Chim. Acta 1990, 233, 41-47.

[17] J. March, Advanced Organic Chemistry, 3rd., Wiley, New York, 1985, p. 906.

Received January 23, 1998. Revision March 29, 1998. 